\title{
Low Temperature Properties of Selected Kramers Rare Earth Oxychlorides.
}

\author{
S. Mat'aš ${ }^{1}$, M. Mihalikik ${ }^{1,2, a}$, B. Klemke ${ }^{1}$, and A. Sokolowski ${ }^{1}$ \\ 1 HZB, Hahn Meitner Platz 1, D-14109 Berlin, Germany \\ 2 IEP SAS, Watsonova 47, 04001 Košice, Slovak Republic
}

\begin{abstract}
In this work, we present low temperature magnetic and electronic properties measured on selected Kramers rare-earth oxychlorides $R E \mathrm{OCl}, R E=\mathrm{Nd}, \mathrm{Gd}$, Dy which adopt the PbFCl-type of structure. Prepared powder samples were characterized by means of standard structural, magnetic and electronic methods as X-ray diffraction $(300 \mathrm{~K})$, heat capacity $(0.3 \mathrm{~K}-12 \mathrm{~K})$ and susceptibility measurements $(2 \mathrm{~K}-300 \mathrm{~K}$, at ambient pressure and hydrostatic pressures up to $0.68 \pm 0.01 \mathrm{GPa}$ ). Our results indicate new transition to the ordered magnetic state for $\mathrm{GdOCl}$ and $\mathrm{NdOCl}$ compound at temperatures of $5 \mathrm{~K}$ and $1.5 \mathrm{~K}$, respectively. We found small increase of magnetization saturation value of dysprosium oxychloride with an applied hydrostatic pressure, but no remarkable changes occur to antiferromagnetic transition temperature $\left(\mathrm{T}_{N} \sim 9.2 \mathrm{~K}\right)$ when a moderate hydrostatic pressure ( $\mathrm{p} \leq 0.68 \pm 0.01 \mathrm{GPa}$ ) was applied. Observed deviations from the Curie Weiss behavior below $26 \mathrm{~K}$ can be caused by the vicinity of the magnetic ordering temperature, or another magnetic effects. The single crystal experiments which will solve this opened question are in progress.
\end{abstract}

\section{Introduction}

Considerable interest was devoted to development, fabrication, and characterization of new phosphorous materials in the past. From among of all, the group of rare earth oxyhalides, especially subgroup oxychlorides, have received considerable attention started early 60ties. It is well known that rare earth oxyhalides exhibit luminiscence phenomena due to the quantum cutting and down conversion. The knowledge of energy level scheme, received mainly from optical measurements, has provided key informations for better understanding this luminiscence phenomena, and for tunning its optical properties. The validity and precision of provided results has been verified directly or indirectly by other techniques as Mössbauer spectroscopy, UV-vis-NIR absorption spectroscopy, magnetic measurements etc. [13]. The representative results were well documented by Hölsä et al. [1]. In his work, the temperature dependencies of avarage paramagnetic susceptibility taken on rare earth oxychlorides were simulated and the results have proved quite good agreement with energy level schemes already proposed on the basis of optical experiments. One should note that deviations between simulated and measured average suceptibilities were at temperatures close to room temperature explained to be the effect of the strong lattice changes.

The low temperature studies of magnetically ordered state of $R E O C l$ materials were not in basic focus. Probably this is the reason why only two members of Kramers (Ce, $\mathrm{Nd}, \mathrm{Sm}, \mathrm{Gd}, \mathrm{Dy}, \mathrm{Er}, \mathrm{Yb}$ ) rare-earth oxychloride group have been identified to possess magnetic ordered state, namely DyOCl with $\mathrm{T}_{N} \sim 11 \mathrm{~K}$ (antiferromagnetic ordering) and $\mathrm{SmOCl}$ with $\mathrm{T}_{N} \sim 8 \mathrm{~K}[1,4]$. To our knowledge, no further direct evidences supporting the magnetic order of other Kramers rare earth oxychloride species have been publis/hed. From structural point of view the $R E O C l$ system for

\footnotetext{
a e-mail: matmihalik@saske.sk
}

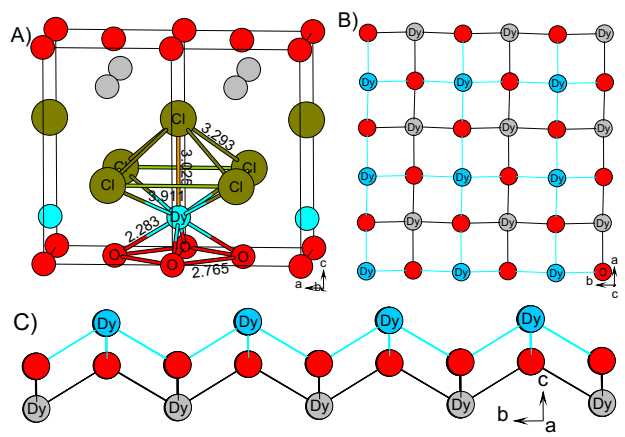

Fig. 1. DyOCl unit cell $(2 \times 1 \times 1)$ and the Dy-O layer seen from [001] and [100] directions. The corresponding color of the element can be deduced from the upper-left-subfigure

lighter rare earth (Kramers) oxychlorides, $R E=\mathrm{Ce}, \mathrm{Nd}$, $\mathrm{Sm}, \mathrm{Gd}$, Dy adopts the tetragonal PbFCl-type of structure $[1,5]$ with the space group $P 4 / n m m$, while for heavier species $\mathrm{RE}=\mathrm{Er}, \mathrm{Yb}$ dimorphism [hexagonal (space group $R \overline{3} \mathrm{~m}$ ) or tetragonal (space group $P 4 / \mathrm{nmm}$ ) type of structure] occurs. The structure of lighter rare earth oxychlorides comprises distinct covalent $(R E O)^{+}$complex cation and anion $\mathrm{Cl}$-layers perpendicular to the $c$-axis of the unit cell. In the Figure 1A two unit cell show rare-earth element neighbors marked with labels and distances (typical for $\mathrm{DyOCl}$ ); the four oxygen atoms placed below and five halogen (chloride) atoms above the rare earth element with one halogen atom in the appical position. The subfigure $1 \mathrm{~B}, 1 \mathrm{C}$ shows the oxygen-rare-earth layer seen from [001] and [100] direction, respectively. Solid circles correspond to oxygen atoms whereas rare earth atoms above and below the oxygen layer are marked with different colors for clarity. 


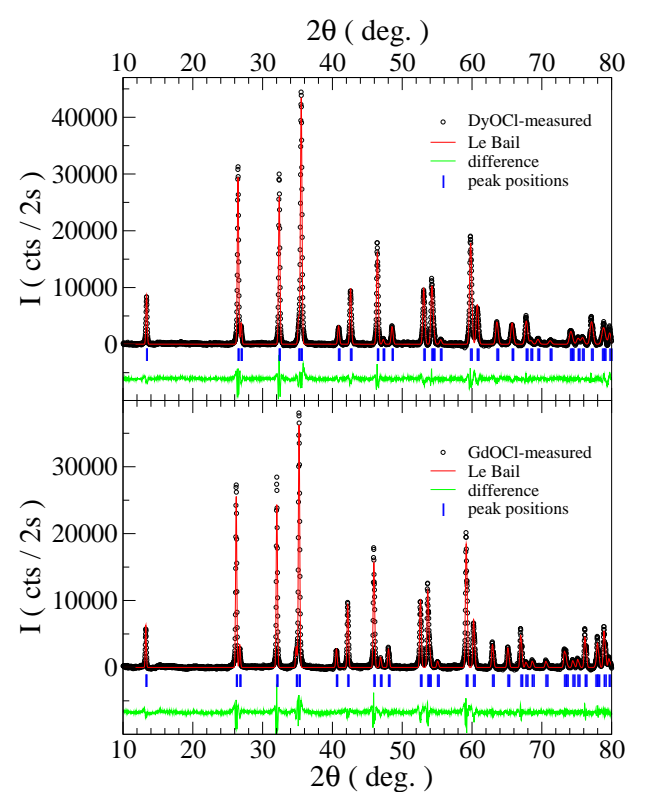

Fig. 2. The X-ray diffraction patterns of $\mathrm{DyOCl}$ (up) and $\mathrm{GdOCl}$ (down) measured at room temperature. The dots correspond to the observed pattern, the solid lines show the calculated pattern using the profile matching mode (Le Bail) and the difference between experimental data and Le Bail fit. The bars show the Bragg reflection positions.

\section{Experimental setup}

Our powder samples were prepared by dissolving the corresponding amount of rare earth oxide (of $99.9 \%$ purity from Sigma-Aldrich co., or Alfa Aesar co. suppliers) in excess of concentrated $\mathrm{HCl}$, then heating in order to evaporate the liquid. The final by-product was annealed in air for 12 hours at $770 \mathrm{~K}$ in alumina crucible. X-ray patterns were recorded on Bruker D8 diffractometer using $\mathrm{Cu}-\mathrm{K}_{\alpha}$ wavelength, performing $\theta-2 \theta$ scan in the range from 10 to 80 degrees (in $2 \theta$ ) at room temperature. The experimental data patterns were refined with help of FullProf program [6] in profile matching mode (Le Bail) for all prepared samples, however, only $\mathrm{DyOCl}$ and $\mathrm{GdOCl}$ patterns are shown in Figure 2. The X-ray refinment results confirmed the single phase character for all prepared oxychloride powders. Our refined lattice parameters are in good agreement with data found in the literature (see Table 1).

Heat capacity measurements were performed in the Laboratory for Magnetic Measurements (LaMMB) in HZB in an Oxford Instruments $14.5 \mathrm{~T}$ cryomagnet equipped with a Heliox ${ }^{3} \mathrm{He}$ insert. Data were collected in a temperature range from $0.3 \mathrm{~K}$ to $2.5 \mathrm{~K}$ in case of $\mathrm{NdOCl}$ sample and

Table 1. The comparison of the lattice parameters of tetragonal (space group $P 4 / \mathrm{nmm}$ ) $R E \mathrm{OCl}$ compounds.

\begin{tabular}{|c|c|c|c|}
\hline & $\mathrm{a}(\mathrm{nm})$ & $\mathrm{c}(\mathrm{nm})$ & ref. \\
\hline \multirow{2}{*}{$\mathrm{NdOCl}$} & $0.40242(1)$ & $0.67737(1)$ & this work \\
& $0.4018(2)$ & $0.6782(4)$ & {$[5]$} \\
\hline \multirow{2}{*}{$\mathrm{GdOCl}$} & $0.39495(1)$ & $0.66678(1)$ & this work \\
& $0.3950(2)$ & $0.6672(4)$ & {$[5]$} \\
\hline \multirow{2}{*}{$\mathrm{DyOCl}$} & $0.39105(1)$ & $0.66228(1)$ & this work \\
& $0.3911(3)$ & $0.6620(6)$ & {$[5]$} \\
\hline
\end{tabular}

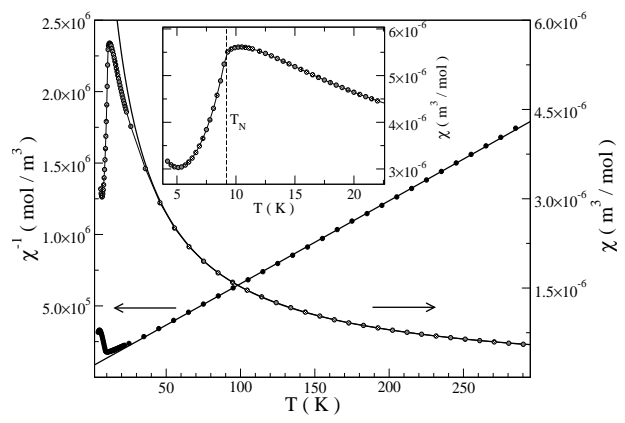

Fig. 3. The molar magnetic susceptibility and the inverse molar magnetic susceptibility measured on DyOCl powder. Lines correspond to best fit to Curie Weiss formula (please, see text for details). The inset shows the molar suseptibility of $\mathrm{DyOCl}$ at low temperatures.

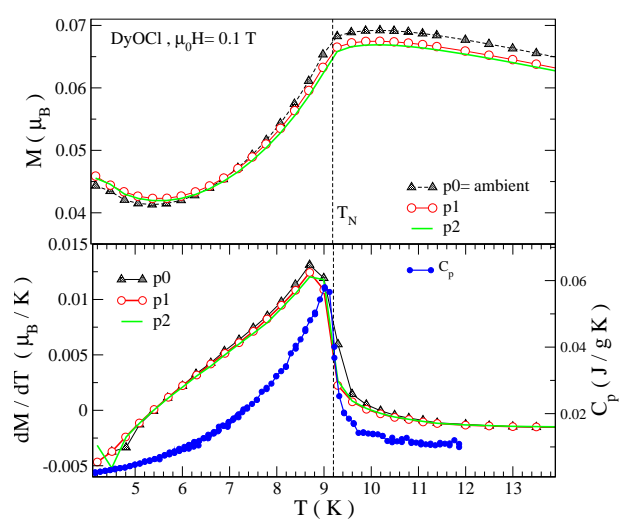

Fig. 4. Magnetic moment of DyOCl powder sample as a function of temperature at ambient $\left(\mathrm{p}_{0}\right)$ and hydrostatic pressures $\mathrm{p}_{1}=0.38 \pm 0.01 \mathrm{GPa}$, and $\mathrm{p}_{2}=0.68 \pm 0.01 \mathrm{GPa}$ (upper Figure). The derivative of magnetic moment at different pressures (down -left scale) and heat capacity $\mathrm{C}_{p}$ of DyOCl powder at ambient pressure (down right scale). Lines are guides to the eyes.

from 4.2 to $12 \mathrm{~K}$ for DyOCl. Both samples were measured in zero magnetic field. The magnetic susceptibility measurements were carried out using a Quantum Design SQUID magnetometer in temperature range from $2 \mathrm{~K}$ up to room temperature for various external magnetic fields up to $5 \mathrm{~T}$. In addition, AC susceptibility measurements were conducted using the ACMS option of Quantum Design Physical Property Measurement System at various temperatures, frequencies and in external magnetic fields up to $7 \mathrm{~T}$.

Magnetic measurements in pressure cell were performed only for DyOCl sample at pressures of $\mathrm{p}_{1}=0.38 \pm$ $0.01 \mathrm{GPa}, \mathrm{p}_{2}=0.68 \pm 0.01 \mathrm{GPa}$ and at ambient pressure $\left(\mathrm{p}_{0}\right)$. We have used the $\mathrm{CuBe}$ piston pressure cell filled with Daphne oil as a pressure transfer medium [7] and superconducting transition of $\mathrm{Pb}$ as an internal pressure sensor.

\section{Results and discussion}

DyOCl: Thermal variations of the molar magnetic susceptibility and inverse molar magnetic susceptibility measured on $\mathrm{DyOCl}$ in magnetic field of $1 \mathrm{~T}$ are displayed in the Figure 3. The solid circles represent experimental points determined from the magnetization measurements. The line represent the best fit to the Curie-Weiss law $\chi(T)$ 


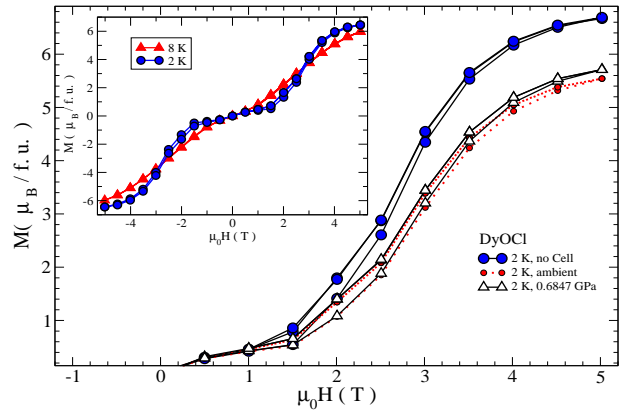

Fig. 5. Magnetization curves measured on $\mathrm{DyOCl}$ powder at $\mathrm{T}=$ $2 \mathrm{~K}$ and $8 \mathrm{~K}$ at ambient and hydrostatic pressure. Lines are guides to the eyes.

$=C /(T-\theta)$ with parameters $\theta=-13.0 \mathrm{~K} \pm 0.3 \mathrm{~K}, C=$ $1.720 \times 10^{-4} \pm 6 \times 10^{-7} \mathrm{~m}^{3} \mathrm{Kmol}^{-1}$ and estimated effective magnetic moment $\mu_{e f f}=10.46 \pm 0.02 \mu_{B}$ which is to compare with $\mathrm{Dy}^{3+}$ free ion value of $10.65 \mu_{B}$. The inset shows in detail a behavior of molar suseptibility at the transition temperature. The deviation from linear dependence of inverse molar susceptibility below $26 \mathrm{~K}$ can be explained by the vicinity of the phase transition, the depopulation of energy levels and/or the magnetic anisotropy of the $\mathrm{Dy}^{3+}$ ion.

Unfortunately, on the basis of our AC measurements of third harmonic susceptibility measured on powder sample (not shown) we were not able to answer, if $\mathrm{DyOCl}$ exhibits the quadrupolar ordering, or not. Therefore new experiments preferably on single crystals become highly desirable.

The magnetic moment of DyOCl powder sample as a function of temperature at ambient $\left(\mathrm{p}_{0}\right)$ and hydrostatic pressures $\mathrm{p}_{1}=0.38 \pm 0.01 \mathrm{GPa}, \mathrm{p}_{2}=0.68 \pm 0.01 \mathrm{GPa}$ is displayed in Figure 4. No remarkable changes at the antiferromagnetic transition temperature of dysprosium oxychloride $\left(\mathrm{T}_{N} \sim 9.2 \mathrm{~K}\right)$ have been detected when a moderate hydrostatic pressure was applied $(\mathrm{p}<0.68 \pm 0.01 \mathrm{GPa})$. The small increase of magnetisation saturation at the magnetic field of $5 \mathrm{~T}$ occurs as the hydrostatic pressure increses to value $0.68 \pm 0.01 \mathrm{GPa}$ (Figure 5). The anomaly in derivative $\mathrm{dM} / \mathrm{dT}$ (Figure 4) associated with the transition temperature $\mathrm{T}_{N}$ does not change the position and corresponds very well with the specific heat lambda-like anomaly at $\mathrm{T}_{N} \sim 9.2 \mathrm{~K}$. The field dependence of an average magnetic moment measured at temeperature of $2 \mathrm{~K}$ and $8 \mathrm{~K}$ (Figure 5) exhibits almost linear behaviour for low fields up to $1.55 \mathrm{~T}$ then, for higher fields, the magnetic signal starts to increase reaching an inflection point at about $3.35 \mathrm{~T}$ and appoaching its saturated value of $6.5 \mu_{B}$ in external field of $5 \mathrm{~T}$. A weak hysteresis in the region between $1.55 \mathrm{~T}$ and $5 \mathrm{~T}$ was observed. As temperature further increases and approaches the transition temperature, field dependence more and more looses its typical S-like shape, approaching almost linear behavior typical for paramagnetic materials.

GdOCl and NdOCl: Molar magnetic susceptibilty and inverse molar susceptibility of $\mathrm{GdOCl}$ are displayed in Figure 6 . The solid circles represent experimental points determined from the magnetization measurements. The best fit to Curie Weiss form result to parameters: $\theta=-10.6 \pm$ $0.1 \mathrm{~K}, C=8.86 \times 10^{-5} \pm 2 \times 10^{-7} \mathrm{~m}^{3} \mathrm{Kmol}^{-1}$ and effective magnetic moment $\mu_{e f f}=7.51 \pm 0.01 \mu_{B}$ which exhibits

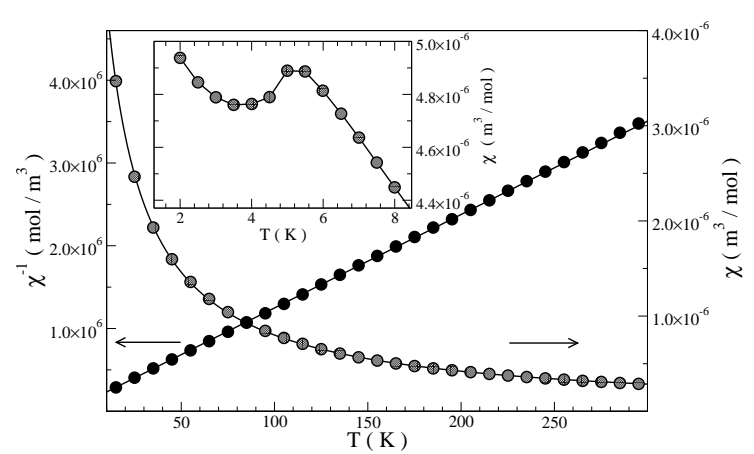

Fig. 6. The thermal variation of the molar magnetic susceptibility and the inverse molar magnetic susceptibility measured on $\mathrm{GdOCl}$ powder. Lines correspond to the best fit to Curie Weiss formula.

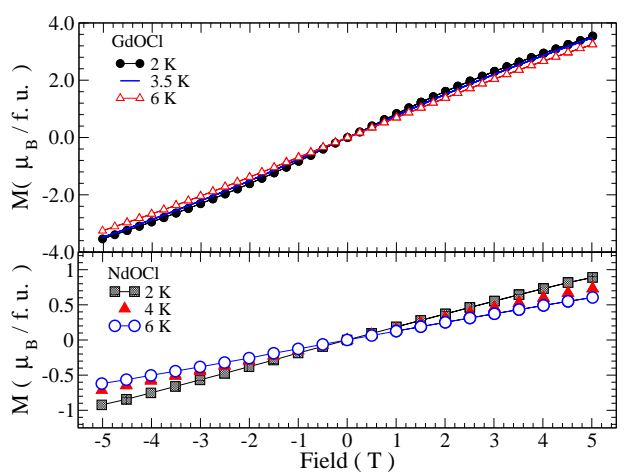

Fig. 7. The field dependence of magnetic moment at different temperatures for $\mathrm{GdOCl}$ and $\mathrm{NdOCl}$.

slightly lower value than moment of free $\mathrm{Gd}^{3+}$ ion $(7.94$ $\left.\mu_{B}\right)$. The inset displays the anomaly in the molar susceptibility at $5 \mathrm{~K}$ which indicates the transition to an ordered state. Our $\mathrm{M}(\mathrm{B})$ curves as measured at temperatures of $2 \mathrm{~K}$, $3.5 \mathrm{~K}$ and $6 \mathrm{~K}$ (Figure 7) do not exhibit hysteretic behavior. At lowest temperature the magnetic moment reaches value of $3.53 \mu_{B}$ in $5 \mathrm{~T}$, however, the field of $5 \mathrm{~T}$ was not strong enough to fully saturate the magnetic signal of GdOCl sample.

In addition, molar magnetic susceptibilty and inverse molar susceptibility of $\mathrm{NdOCl}$ are presented in the Figure 8 . Here again the solid circles represent experimental data points. The best fit to Curie Weiss formula $\chi(T)=$ $C /(T-\theta)$ gives fitting parameters $\theta=-28.7 \pm 0.3 \mathrm{~K}, C$ $=1.840 \times 10^{-5} \pm 6 \times 10^{-8} \mathrm{~m}^{3} \mathrm{Kmol}^{-1}$. The estimated effective magnetic moment of value $\mu_{\text {eff }}=3.42 \pm 0.01 \mu_{B}$ is to compare to $\mathrm{Nd}^{3+}$ free ion value of $3.61 \mu_{B}$.

The linear dependence of inverse molar susceptibility of $\mathrm{NdOCl}$ is described very well in the range from $25 \mathrm{~K}$ to $295 \mathrm{~K}$, however, both $i$ ) the deviation from linear dependence below $25 \mathrm{~K}$ and $i$ ) Curie parameter $\theta_{C W}=-28.7 \mathrm{~K}$ indicate possible phase transition at low temperatures. Since the limitation of our experimental apparatus for magnetization measurements did not allow us to perform experiments at temperatures below $1.8 \mathrm{~K}$, we have used heat capacity technique to probe phase transitions at low temperatures. Indeed, in spite of our limitations in determination of magnetic properties to high temperature region ( $\mathrm{T}$ $>1.8 \mathrm{~K}$ ), the heat capacity technique, capable to probe cooperative transitions at low temperature, undoubtedly con- 


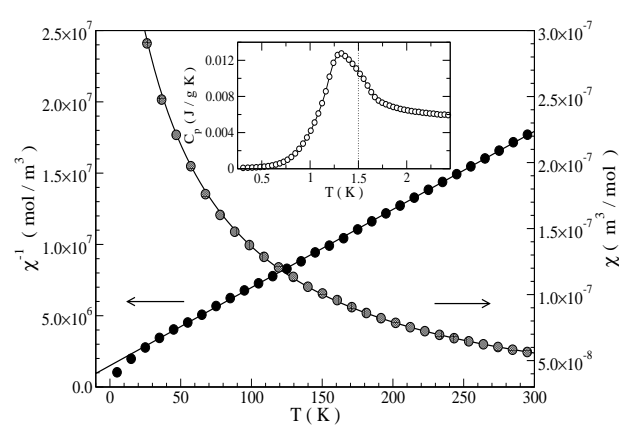

Fig. 8. The thermal variation of the molar magnetic susceptibility and the inverse molar magnetic susceptibility taken on $\mathrm{NdOCl}$ powder. Lines correspond to the best fit to Curie Weiss formula (please, see text for details). Inset shows the lambda like anomaly at transition temperature $\mathrm{T}_{N} \sim 1.5 \mathrm{~K}$ in the heat capacity.

firmed the lambda-like anomaly of $\mathrm{NdOCl}$ sample at temperature $\sim 1.5 \mathrm{~K}$ (see inset of Figure 8 ). This perfectly resemble the anomaly of antiferromagnetic DyOCl system as presented in Figure 4. Field dependencies of magnetic moment are taken only in the paramagnetic region at temperatures of $2 \mathrm{~K}, 4 \mathrm{~K}$ and $6 \mathrm{~K}$, therefore show simple linear like behavior (see Figure 7).

\section{Conclusions}

The low temperature specific heat and magnetic properties measured on several members of Kramers rare earth oxychlorides $R E \mathrm{OCl}, R E=\mathrm{Dy}, \mathrm{Gd}, \mathrm{Nd}$ have shown two new transition temperatures to ordered state at $1.5 \mathrm{~K}$ and $5 \mathrm{~K}$ for $\mathrm{NdOCl}$ and $\mathrm{GdOCl}$ compounds, respectively. Our hydrostatic pressure study performed on dysprosium oxychloride shows no remarkable changes to an antiferromagnetic transition temperature $\mathrm{T}_{N} \sim 9.2 \mathrm{~K}$ with applied hydrostatic pressure. Only a small increase of saturation magnetisation has been induced by applying pressure of $0.68 \mathrm{GPa}$. Observed deviations from the Curie Weiss behavior for Dy$\mathrm{OCl}$ sample below $26 \mathrm{~K}$ might be caused by the vicinity of the phase transition, the depopulation of energy levels and/or the magnetic anisotropy of the $\mathrm{Dy}^{3+}$ ion. However, the possibility of ongoing quadrupolar order was not completely ruled out. This however, can be resolved when appropriate single crystals of $\mathrm{DyOCl}$ will be available.

Acknowledgement: S. M. and M. M. would like to acknowledge the support of LaMMB at the Helmholtz-Zentrum Berlin. M. M. also acknowledges support from European social fund under contract 26110230034.

\section{References}

1. J. Hölsä, R. J. Lamminmäki, M. Lastusaari, P. Porcher, R. Sáez-Puche, J. All. \& Comp. 303-304, 498 (2000)

2. J. M. Friedt, J. Mac Cordick, J. P. Sanchez, Inorg. Chem. 22, 2910 (1983)

3. J. Hölsä, R. J. Lamminmäki, P. Porcher, P. Derén, W.Str ek, Spectroch. Act. A 54, 2189 (1998)

4. D. Elmaleh, D. Fruchart, J. C. Joubert, J. de Phys. 32, C1-741 (1971)
5. D. H. Templeton, C. H. Dauben, J. Am. Chem. Soc. 75, 6069 (1953)

6. T. Roisnel, J. Rodriguez-Carvajal, Mater. Sci. Forum 378, 118 (2001)

7. J. Kamarád, Z. Machátová, Z. Arnold, Rev. Sci. Instrum. 75, 5022 (2004)

8. R. M. Galéra, E. Sole, M.Amara, P. Morin, P. Burlet, A. P. Murani, J. Phys.: Condens. Matter 15, 6269 (2003)

9. E. Matsuoka, T. Tayama, T. Sakibara, Z. Hiroi, N. Shirikawa, N. Takeda and M. Ishikawa, J. Phys. Soc. Jpn 76, 084717 (2007) 